\title{
Dynamics of resonantly interacting equatorial waves
}

\author{
By CARLOS F. M. RAUPP* and PEDRO L. SILVA DIAS, Department of Atmospheric Sciences, \\ Institute of Astronomy, Geophysics and Atmospheric Sciences, University of São Paulo, São Paulo/SP, Brazil
}

(Manuscript received 24 August 2004; in final form 30 April 2005)

\begin{abstract}
In this paper we explore some dynamical features on the non-linear interactions among equatorial waves. The shallowwater equation model with the equatorial $\beta$-plane approximation is used for this purpose. The Galerkin method is applied to the governing equations with the basis functions given by the eigensolutions of the linear problem. From the phase space expansion of two particular integrals of motion of the system, quadratic to lowest order, some constraints are obtained which the coupling coefficients must satisfy in order to ensure the invariance of such integrals. From the numerical evaluation of the coupling coefficients, these constraints are used to determine the possible resonant triads among equatorial waves. Numerical integrations of the resonant three-wave problem show that the energy of the waves in a resonant triad evolves periodically in time, with the period and amplitude of the energy oscillations dependent on the magnitude of the initial amplitudes of the waves and the way in which the initial energy is distributed among the triad components. The high-frequency modes are found to be energetically more active than the low-frequency modes. The latter tend to act as 'catalytic' components in a resonant triad. Integrations of the problem of two resonant triads coupled by a single mode point out the importance of gravity waves in the intertriad energy exchanges, suggesting the significance of these modes in the redistribution of energy throughout the atmospheric motion spectrum. The results also show that the intertriad energy exchanges provided by the highest frequency mode of two triads occur in a longer time-scale than the intratriad interactions. Therefore, these results also suggest the importance of the high-frequency modes in the generation of the low-frequency variability (intraseasonal and even longer term) of the atmospheric flow.
\end{abstract}

\section{Introduction}

The equatorial trapping of large-scale wave-like disturbances is a prominent characteristic of the atmospheric circulation. This phenomenon was theoretically discovered by Matsuno (1966), who derived a complete set of linear wave-mode solutions of the shallow-water equations on the equatorial $\beta$-plane. After this theoretical finding, several studies have provided observational evidence of the existence of these waves in the equatorial atmosphere (Yanai and Maruyama, 1966; Maruyama, 1967; Gruber, 1974; Zangvil and Yanai, 1980; Takayabu, 1994; Dunkerton and Baldwin, 1995; Magaña and Yanai, 1995; Pires et al., 1997; Wheeler and Kiladis, 1999). As a consequence, the equatorially trapped waves have been applied in explaining fundamental features of tropical climate. The phenomena that have been explained in terms of equatorial wave theory include HadleyWalker circulation (Webster, 1972; Gill, 1980; Lau and Lim,

\footnotetext{
* Corresponding author.

e-mail: cfmraupp@model.iag.usp.br

Postal address: Rua do Matão, 1226, Cidade Universitária, São PauloSP, Brazil 05508-090.

DOI: $10.1111 / \mathrm{j} .1600-0870.2006 .00151 . x$
}

1982; Lim and Chang, 1983), atmospheric teleconnection patterns (Lim and Chang, 1983; Raupp and Silva Dias, 2004), the low-frequency oscillation initially observed by Madden and Julian (1972) (Lau and Peng, 1987; Wang and Rui, 1990) and the El Niño Southern Oscillation (Lau, 1981; Hirst, 1986).

However, although a significant advance has been obtained on equatorial waves, especially on their interaction with moist convection, the non-linear behavior is far from completely understood. Particularly, most theoretical studies are based on linearized equations about simplified basic states and the non-linear dynamics of equatorial waves needs to be better understood. Despite the observational evidence of wave-wave interactions in the tropics (Kanamitsu et al., 1972), not many analytical works have been published on this matter. Domaracki and Loesch (1977) first studied resonant triads of equatorial waves using the asymptotic method of multiple scales. They obtained energy solutions which show that the triad member having the maximum absolute frequency always grows (or decays) at the expense of the other two triad members. Loesch and Deininger (1979) extended the results of Domaracki and Loesch for resonantly interacting waves in coupled triad configurations and also pointed out the importance of the maximum absolute frequency modes in individual triads for energy transfers throughout the system. 
On the other limit of energy scale, Salmon (1980) extended the results of geostrophic turbulence (Charney, 1971) (mainly, the concepts of energy and enstrophy conservation) to low latitudes.

Ripa (1981) formulated the non-linear wave-wave interaction problem for barotropic non-divergent Rossby waves and internal gravity waves on a vertical plane. Ripa applied Galerkin formalism with the basis functions given by the eigensolutions of the linear problem and showed that the conservation of two particular integrals of motion, quadratic to lowest order, leads to interesting properties that the coupling coefficients must satisfy in order to ensure the invariance of such integrals. This formalism was extended to the equatorial wave-guide in Ripa (1982, 1983a,b). Recently, Majda et al. (1999) analyzed the role of topography in resonantly coupling equatorial waves. They used an asymptotic expansion in terms of multiple scales and showed that by resonance with topography the equatorial waves can also interact with each other and exchange energy. Especially, the Kelvin wave can excite large-scale Yanai and Rossby waves with specific zonal wavelengths and slow down its phase speed by this resonance mechanism.

A straightforward view of Ripa's formalism will be carried out here in order to organize the theoretical framework. In this paper we used the results of Ripa's formalism to determine the possible resonant triads among equatorial waves and analyzed their dynamics by numerical integrations of the model equations in the phase space. The main concern here is to highlight the implications of the results for tropical atmospheric dynamics. It shall be shown that the resonant energy exchanges among equatorial waves have interesting implications for tropical atmospheric dynamics. Particularly, the results of the numerical integrations of the problem of two resonant triads coupled by a single mode suggest the importance of gravity waves (and, thus, moist convection) in the generation of the low-frequency (intraseasonal and/or even longer term) variability of the atmospheric circulation. Thus, as shall be discussed in Section 6, this paper provides an alternative explanation for the origin of intraseasonal oscillations in the tropics. In Section 2, the governing equations, the spectral method of solution and the concept of resonance in non-linear interactions are presented. In Section 3 we describe a straightforward view of Ripa's formalism on non-linear wave-wave interactions on the equatorial wave-guide. In Section 4 the main result of this formalism is used to determine the possible resonant triads among equatorial waves by numerical computation of the coupling coefficients. In Section 5 we explore the dynamics of the energy exchanges among equatorial waves in resonant triads by numerical integrations of the model equations in spectral space. The results of both the three-wave problem and the problem of two triads coupled by a single mode are shown. A summary of the results obtained in the previous sections and the implications of these results for the tropical atmospheric dynamics are presented in Section 6.

\section{Model equations and solution method}

The shallow-water model on the equatorial $\beta$-plane adopted in the present work can be described by the following system of equations in the vector form:

$\frac{\partial \xi}{\partial t}+\Omega \xi=N$

System (2.1) is shown after normalization, using the scales $[L]=(c / \beta)^{1 / 2}$ and $[T]=(1 / c \beta)^{1 / 2}$ of length and time, respectively, and $h_{0}$ as the vertical scale, where $\xi=[u(x, y, t), v(x, y$, $t), \phi(x, y, t)]^{\mathrm{T}}$ is the state vector of the model and

$$
\begin{gathered}
\left.\Omega=\left[\begin{array}{ccc}
0 & -y & \frac{\partial}{\partial x} \\
y & 0 & \frac{\partial}{\partial y} \\
\frac{\partial}{\partial x} & \frac{\partial}{\partial y} & 0
\end{array}\right] \quad \begin{array}{c}
\text { and } \\
-\left(u \frac{\partial u}{\partial x}+v \frac{\partial u}{\partial y}\right) \\
-\left(u \frac{\partial v}{\partial x}+v \frac{\partial v}{\partial y}\right) \\
N= \\
-\left(u \frac{\partial \phi}{\partial x}+v \frac{\partial \phi}{\partial y}\right)-\phi\left(\frac{\partial u}{\partial x}+\frac{\partial v}{\partial y}\right)
\end{array}\right]
\end{gathered}
$$

are the linear operator associated with the linearized equations and the non-linear term, respectively. Here, $u$ and $v$ are, respectively, the components of the two-dimensional velocity field on the $x$ (eastward) and $y$ (northward) directions; $\phi$ is the geopotential field of the free surface, $c=\sqrt{g h_{0}}$ is the phase speed of pure gravity waves, $h_{0}$ is the equivalent depth and $g$ is the effective gravity acceleration. The parameter $\beta=\mathrm{d} f / \mathrm{d} y$ is the Rossby parameter (where $f$ is the Coriolis parameter) and is assumed here as a constant and with its equatorial value.

System (2.1) is an appropriate model for describing atmospheric and oceanic motions with somewhat long time-scales and whose horizontal length-scales are relatively larger than their vertical ones, that is, for describing phenomena that do not depend crucially on temporal changes of the vertical density stratification. In the linear context, the shallow-water equations can also be regarded as the governing equations for the time evolution of the horizontal structure associated with a particular internal vertical mode of the upper-lidded primitive equations (Silva Dias et al., 1983). In this context, the equivalent depth $h_{0}$ is the separation constant and is obtained as an eigenvalue of the vertical structure equation. However, as far as the non-linear terms are restored this correspondence is no longer valid, because in this case the Galerkin projection of the three-dimensional primitive equations onto a particular vertical mode does not fall naturally into the non-linear shallow-water equations given by eqs. (2.1) and (2.2). As a consequence, caution is necessary in interpreting the results of this paper for the fully vertically stratified atmosphere, as shall be mentioned in Section 6 . 
Zonal periodicity in the $x$-direction and bounded solution as $|y|$ goes to infinity provide the boundary conditions for system (2.1). These boundary conditions define the eigenfunctions of the linear operator $\Omega$ as Hermite functions in the $y$-direction multiplied by trigonometric functions in the $x$-direction. Such eigenmodes form an orthogonal and complete set and are used as the basis functions in the expansion of $\xi$ in a series as in

$\xi(x, y, t)=\sum_{a} c_{a}(t) \xi_{a}$

where $c_{a}(t)$ are the spectral coefficients defined as the inner product between the state vector and a particular eigenfunction $\xi_{a}$. The subscript $a$ characterizes a particular expansion mode and is given by $a=(k, n, r)$, where $k$ is the zonal wavenumber, $n$ is the meridional mode, which distinguishes the meridional structure of the eigenfunctions; $r=1$ for Rossby waves (RW), $r=2$ for inertio-gravity waves propagating westward (WGW) and $r=3$ for inertio-gravity waves propagating eastward (EGW). The mixed Rossby-gravity waves (MRGW) correspond to the $n=0$ mode and are included in either $r=1$ (for $k>2^{-1 / 2}$ ) or $r$ $=2\left(\right.$ for $\left.k<2^{-1 / 2}\right)$ categories. The Kelvin wave is represented by $n=-1$ and $r=3$. The determination of the eigenfunctions of $\Omega$ and a complete discussion on the properties of such free linear wave solutions of the governing equations are found in Matsuno (1966).

For each expansion mode with parameters $\left(\omega_{a}, k_{a}, n_{a}, r_{a}\right)$, where $\omega_{a}$ are the eigenfrequencies associated with the eigenfunctions $\xi_{a}$, and the structure functions $\xi_{a}$, there is another mode, with parameters $\left(-\omega_{a},-k_{a}, n_{a}, r_{a}\right)$ and structure functions $-\xi_{a}^{*}$, where the superscript $*$ indicates the conjugate complex; the latter is denoted by $a^{*}$ and called as the conjugate mode of $a$. Two conjugate modes are mathematically independent, in the sense that the expansion (2.3) must consider both components of each conjugate pair $\left(a, a^{*}\right)$, but physically equivalent because the reality of the state variables $u, v$ and $\phi$ in eq. (2.3) implies that

$c_{a^{*}}=-c_{a}^{*}\left(\omega_{a^{*}}=-\omega_{a}, k_{a^{*}}=-k_{a}\right)$.

The expansion (2.3) is an exact solution of eq. (2.1) provided that the spectral coefficients satisfy the following system of ordinary differential equations

$\frac{\mathrm{d} c_{a}(t)}{\mathrm{d} t}-\mathrm{i} \omega_{a} c_{a}(t)=\sum_{b} \sum_{c} \sigma_{a}^{b c} c_{b}^{*}(t) c_{c}^{*}(t)$.

In eq. (2.5) above, $\sigma_{a}^{b c}$ represent the coupling coefficients among three particular modes $a, b$ and $c$, and are given by

$$
\begin{aligned}
\sigma_{a}^{b c}= & \left\langle\left[ u_{b} \mathrm{i} k_{c} \xi_{c}+v_{b} \frac{\partial \xi_{c}}{\partial y}+(0,0,1)^{\mathrm{T}} \phi_{b}\right.\right. \\
& \left.\left.\times\left(\mathrm{i} k_{c} u_{c}+\frac{\partial v_{c}}{\partial y}\right)+C P\right] \cdot \xi_{a}\right\rangle \delta_{a b c} .
\end{aligned}
$$

In eq. (2.6) above, $\langle\cdot\rangle$ represents the inner product given by

$$
\begin{aligned}
\langle p(x, y) \cdot q(x, y)\rangle= & \int_{-L_{x}}^{L_{x}} \int_{-\infty}^{+\infty}\left(p_{1} q_{1}^{*}+p_{2} q_{2}^{*}+p_{3} q_{3}^{*}\right) \\
& \times \mathrm{d} y \mathrm{~d} x
\end{aligned}
$$

where $p$ and $q$ are arbitrary three-dimensional vector fields satisfying the boundary conditions of model (2.1) and the subscripts 1,2 and 3 refer to their scalar components; $L_{x}$ corresponds to the zonal period in dimensionless units and the superscript ' $\mathrm{T}$ ' in eq. (2.6) refers to the transposition operation so that $(0,0,1)^{\mathrm{T}}$ corresponds to the unit vector in the vertical direction. The term $C P$ in eq. (2.6) means cyclical permutations between the superscripts $b c$ and, thus, the coupling coefficients $\sigma_{a}^{b c}$ are invariant under permutations of these indices. For a suitable normalization of the eigenfunctions $\xi_{a}$, the coupling coefficients are real. From eqs. (2.5) and (2.6) it can be noted that the non-linear interactions take place in triad configurations in which the coupling coefficients represent a measure of how far the product between two particular modes b and c can project onto another particular mode a. Thus, all information on the non-linearity of the model is contained in these coefficients. The coefficients $\delta_{a b c}$ represent the interaction condition for the triad $(\mathrm{a}, \mathrm{b}, \mathrm{c})$ and are given by

$\delta_{a b c}=\left\{\begin{array}{l}2 L_{x} \delta\left(k_{a}+k_{b}+k_{c}\right) \text { if } n_{a}+n_{b}+n_{c}=\text { odd } \\ 0 \quad \text { otherwise }\end{array}\right.$

where the delta function in eq. (2.8a) results from the orthogonality of the trigonometric functions. It is well known (Phillips, 1960; Bretherton, 1964; Ripa, 1981, 1982, 1983a,b) that if the amplitude is small such that, to a first approximation, the evolution of the system is controlled by the linear part of eq. (2.5), only the interacting triads whose components satisfy the relation

$\omega_{a}+\omega_{b}+\omega_{c}=0$

contribute significantly to the non-linearity of the model. These triads whose components satisfy the interaction condition (2.8a) for $k_{a}+k_{b}+k_{c}=0$ and the resonance condition (2.9) are usually referred to as resonant triads.

This interesting phenomenon in non-linear wave-wave interactions was noticed by Phillips (1960) while discussing the role of small non-linear terms in the theory of ocean waves and has then been applied to a wide range of problems in physics. A rich and complete discussion on resonance in wave-wave interactions can be found in Bretherton (1964) in his analysis of a simple wave equation forced by a quadratic term. In the equatorial wave spectrum is a variety of resonant triads, as will be shown in Section 4. It is also important to mention that coupled resonant triads can also exist in the spectrum of the equatorial waves, i.e. resonant interactions involving a larger number of modes, as discussed later.

Therefore, knowledge of the possible resonant triads involving equatorial waves is of great importance for the tropical atmospheric dynamics, because, a priori, these triads determine the 
non-linear interactions in the spectrum of the equatorial waves. The next sections aim to analyze the time evolution of the mode energies in resonant triads. This analysis is no longer obvious and requires a further investigation on the internal dynamics of the model (2.1). This is exactly what shall be done in the next sections. Some constraints that the coupling coefficients must satisfy will be discussed in the next section. These are conditions that the coupling coefficients must satisfy as a consequence of the invariance of two particular integrals of motion, following the mathematical formalism of Ripa (1982, 1983a). As will be shown, total energy and pseudo-momentum conservations lead to interesting properties that determine the time evolution of the modal amplitudes in a resonant triad and thus interesting implications for the dynamics of equatorial waves.

\section{Integral constraints}

System (2.1) is constrained by the existence of several integrals of motion. Two (and only two) of them have, to lowest order, a quadratic dependence in terms of the dependent variables of the model. The consequences of their conservation to the evolution of (2.1) in the phase space are more powerful than the consequences of the other conservation laws. These quadratic to lowest-order integrals of motion are the total energy and the total pseudo-momentum defined by their respective equations in conservative forms:

$$
\begin{aligned}
& \frac{\partial E}{\partial t}+\nabla \cdot\left[\vec{V}\left(E+\frac{1}{2} \phi^{2}\right)\right]=0 \\
& \frac{\partial P}{\partial t}+\nabla \cdot[\vec{V}(P+u)]+\frac{1}{2} \frac{\partial}{\partial x}\left[\phi^{2}-\left(u^{2}+v^{2}\right)\right]=0 .
\end{aligned}
$$

In the equations above, the superscript ' $T$ ' corresponds to the transposition operation so that $\vec{V}=(u, v)^{\mathrm{T}}$ refers to the twodimensional velocity field vector. The variables $E$ and $P$ are, respectively, the energy and pseudo-momentum densities defined by

$E=\frac{1}{2}\left[\left(u^{2}+v^{2}+\phi^{2}\right)+\phi\left(u^{2}+v^{2}\right)\right]$

$P=\phi u-\frac{1+\phi}{2}(q-y)^{2}$

where $q$ is the potential vorticity and $y$ refers to the dimensionless Coriolis parameter.

The theoretical framework employed in this work follows the mathematical formalism of Ripa (1983a) and is also presented in Section 3 of Raupp and Silva Dias (2005). Thus, further details of this formalism will not be repeated here. The most important result for the purpose of the next sections is that, as a consequence of the conservation of total energy and pseudo-momentum, if a certain triad (a, b, c) satisfies the resonance condition (2.9) the coupling coefficients associated with this particular triad satisfy the relations

$\sigma_{a}^{b c}+\sigma_{b}^{a c}+\sigma_{c}^{a b}=0$

$s_{a} \sigma_{a}^{b c}+s_{b} \sigma_{b}^{a c}+s_{c} \sigma_{c}^{a b}=0$.

The parameter $s$ in eq. (3.2b) above is the slowness index (Ripa, 1981, 1982, 1983a,b), which is defined as the inverse of the zonal phase speed of the waves. Condition (3.2a) is a consequence of the conservation of total energy, whereas relation (3.2b) results from the pseudo-momentum conservation. These constraints have some interesting implications for the dynamics of the equatorial waves. The immediate consequence of eqs. (3.2a) and (3.2b) is that resonant interactions conserve the quadratic (lowest-order) part of total energy and pseudomomentum; the changes of $O\left(c^{3}\right)$ terms are owing to interactions by off-resonant triads. From eqs. (3.2a) and (3.2b) it can also be noted that, in a resonant triad, the component which has the coupling coefficient with the opposite sign of the other two (and, consequently, with the greatest absolute value) has the intermediate slowness. Thus, in this kind of interaction, the wave with the intermediate slowness always gains energy from (or releases energy to) the other two. Furthermore, using the definition of the slowness $s$ (inverse of the phase speed of the waves) and the interaction condition $\left(k_{a}+k_{b}+k_{c}=0\right)$, it is easy to show that the wave with the intermediate slowness in a certain resonant triad has the largest absolute frequency of the triad, i.e.

$\frac{\sigma_{a}^{b c}}{\omega_{a}}=\frac{\sigma_{b}^{a c}}{\omega_{b}}=\frac{\sigma_{c}^{a b}}{\omega_{c}}=\gamma_{a b c}$.

Therefore, in a resonant triad the wave with the largest absolute frequency is the most energetically active, that is, always gains energy from (or releases energy to) the other two components. Moreover, eq. (3.3) also shows that high-frequency modes in a resonant triad are energetically more active than the lowfrequency modes. As will be shown in the next sections, this constraint has an important implication not only in the determination of the possible resonant interactions but also in the time evolution of the wave energies in such interactions.

\section{Determination of the possible resonant triads among equatorial waves}

In this section we determine the possible resonant triads involving equatorial waves. The possible resonant triads were determined by the numerical calculation of the coupling coefficients in eq. (2.6) and by using the constraint (3.2a). For the numerical computation of the coupling coefficients in eq. (2.6) we used the Gauss-Hermite quadrature formula. It is important to mention that, because eq. (2.1) allows only a discrete number of normal modes, the resonance condition (2.9) cannot be exactly satisfied in practice. Thus, a certain tolerance $\Delta$ in eq. (3.2a) is needed in 
Table 1. Possible resonant triads involving equatorial waves for $h_{0}=250 \mathrm{~m}$. The table shows, from left to right, the triad components and their respective eigenfrequencies and coupling coefficients. The modes are characterized, from left to right, by the zonal wavenumber, the meridional mode and the wave type

\begin{tabular}{|c|c|c|c|c|c|c|c|c|c|}
\hline & $a$ & $b$ & $c$ & $\omega_{a}$ & $\omega_{b}$ & $\omega_{c}$ & $\sigma_{a}^{b c}$ & $\sigma_{b}^{a c}$ & $\sigma_{c}^{a \mathrm{~b}}$ \\
\hline 1 & $0,-1, \mathrm{~K}$ & $1,1, \mathrm{WG}$ & $1,1, \mathrm{EG}$ & 0 & 1.71 & -1.78 & 0 & -0.1225 & 0.12765 \\
\hline 2 & $1,1, \mathrm{R}$ & $3,3, \mathrm{WG}$ & 4,3,EG & 0.0758 & 2.687 & -2.86 & 0.00607 & 0.04377 & -0.0519 \\
\hline 3 & $5,1, \mathrm{R}$ & 1,3,EG & $6,3, \mathrm{WG}$ & 0.2711 & -2.67 & 2.906 & 0.012614 & 0.054344 & -0.0631 \\
\hline 4 & $0,0, \mathrm{WG}$ & $4,1, \mathrm{WG}$ & 4,2,EG & 1 & 1.83 & -2.49 & -0.028472 & -0.064398 & 0.0909 \\
\hline 5 & $0,1, \mathrm{R}$ & $5,2, \mathrm{R}$ & $5,0, \mathrm{M}$ & 0 & 0.183 & 0.577 & 0 & -0.07624 & 0.07588 \\
\hline 6 & $7,1, \mathrm{R}$ & $6,-1, \mathrm{~K}$ & 1,3,EG & 0.292 & -1.39 & -2.67 & -0.00625 & -0.049643 & 0.05715 \\
\hline 7 & $5,-1, \mathrm{~K}$ & 2,1,EG & 7,1,WG & -1.156 & -1.86 & 2.21 & 0.01684 & 0.076963 & -0.0914 \\
\hline 8 & 1,2,EG & $3,1, \mathrm{WG}$ & $4,0, \mathrm{M}$ & -2.27 & 1.756 & 0.64 & 0.09563 & -0.0816 & -0.0251 \\
\hline 9 & $5,0, \mathrm{M}$ & $1,2, \mathrm{WG}$ & 4,3,EG & 0.577 & 2.225 & -2.86 & -0.0185 & -0.08019 & 0.10136 \\
\hline 10 & $2,-1, \mathrm{~K}$ & $7,0, \mathrm{M}$ & $5,2, \mathrm{EG}$ & -0.462 & 0.477 & -2.6 & 0.03389 & 0.007342 & -0.04 \\
\hline
\end{tabular}

order to find the possible resonant interactions. The number of resonant triads found by condition (3.2a) depends on the tolerance and, through the fundamental wavenumber, on the equivalent depth $h_{0}$. However, although the choice of the tolerance $\Delta$ and the equivalent height $h_{0}$ influence the size of the resonance spectrum, it was found that the resonant triads are highly self-consistent, i.e. exhibit a remarkably similar composition irrespective of $h_{0}$ and the choice of $\Delta$. Therefore, for an illustrative purpose, only unique values of $h_{0}(250 \mathrm{~m})$ and $\Delta\left(10^{-2}\right)$ are discussed here (Table 1). This value of $h_{0}$ is associated with the dominant vertical mode for the atmospheric flow in the tropical region (Silva Dias and Bonatti, 1985), because it corresponds to the mostly excited vertical mode by the typical heating resultant from the organized moist convection in the tropics (DeMaria, 1985). Table 1 shows the mode components of each triad and their correspondent eigenfrequencies and coupling coefficients. It is important to state that Table 1 presents only some examples of the main kinds of resonant triads found. Obviously, there are more triads that are not shown in Table 1 but these triads have the same kind of waves as those shown in Table 1.

Table 1 shows that, in general, the resonant triads obtained involve all the equatorial wave types. They are strongly dominated by triads with two high-frequency modes (Kelvin or inertiogravity) and one low-frequency mode (Rossby or mixed Rossbygravity). Some strictly low-frequency (case 5 of Table 1) and strictly high-frequency triads (cases 4 and 7) also exist, but no triads involving solely the inertio-gravity modes, satisfying condition (3.2a), could be found.

From Table 1 it can be noted that the coupling coefficients in a resonant triad involving equatorial waves are indeed proportional to the individual absolute frequencies of the wave components. As a consequence, in a resonant interaction involving two inertio-gravity modes and one Rossby mode the coupling coefficient associated with the Rossby mode is much smaller in absolute value than the coupling coefficients associated with the inertio-gravity modes. As a result, this kind of interaction is characterized by a catalytic energy exchange between the two high-frequency modes, while the Rossby wave energy remains almost unchanged in time, as will be shown in the next section. Especially, in resonant triads containing the zonally symmetric Rossby or Kelvin modes, as a consequence of the zero-frequency of these modes, their coupling coefficients are zero and thus their energy remains completely unaltered in a resonant interaction.

Loesch and Deininger (1979) determined the resonant triads among equatorial waves using the conditions (2.8a) and (2.9), together with the dispersion relation, following Longuet-Higgins and Gill (1967). In general, the resonant interactions found by Loesch and Deininger are in agreement with those obtained here.

\section{Dynamics of resonant interactions among equatorial waves}

Possible resonant interactions involving equatorial waves were discussed in the previous section. As, in a first approximation, only these triads have a significant role on the non-linear evolution of eq. (2.5), besides the knowledge of the possible resonant triads it is also interesting to analyze the triad dynamics. The dynamics of the resonant three-wave problem is discussed in Section 5.1, i.e. system (2.5) is truncated to consider only three waves satisfying the conditions (2.8a) for $k_{a}+k_{b}+k_{c}=0$ and (3.2a). The problem of two resonant triads coupled by a single mode is discussed in Section 5.2.

\subsection{Resonant three-wave problem}

The resonant three-wave problem has the following formulation in the phase space:

$$
\begin{aligned}
& \frac{\mathrm{d} c_{1}}{\mathrm{~d} t}-\mathrm{i} \omega_{1} c_{1}=\sigma_{1}^{23} c_{2}^{*} c_{3}^{*} \\
& \frac{\mathrm{d} c_{2}}{\mathrm{~d} t}-\mathrm{i} \omega_{2} c_{2}=\sigma_{2}^{13} c_{1}^{*} c_{3}^{*} \\
& \frac{\mathrm{d} c_{3}}{\mathrm{~d} t}-\mathrm{i} \omega_{3} c_{3}=\sigma_{3}^{12} c_{1}^{*} c_{2}^{*} .
\end{aligned}
$$




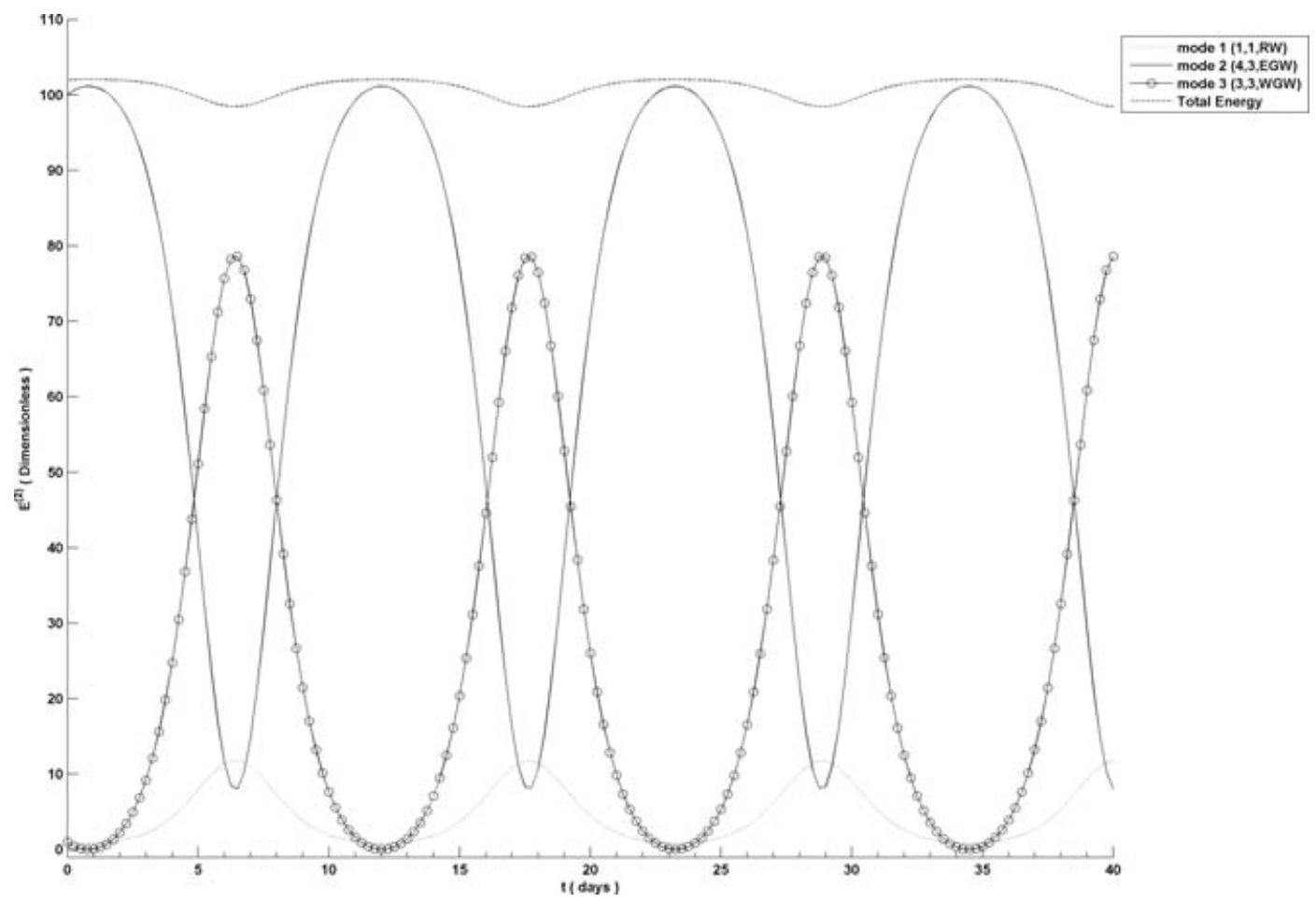

Fig. 1. Time evolution of the modal quadratic energies associated with the solution of the resonant three-wave problem for the triad composed of a Rossby wave (RW) with $k=1$ and $n=1$ (mode 1), an inertio-gravity wave propagating eastward (EGW) with $k=4$ and $n=3$ (mode 2 ) and an inertio-gravity wave propagating westward (WGW) with $k=3$ and $n=3$ (mode 3). The total quadratic energy given by the sum of the energy of the individual components is also displayed. The initial amplitudes of the triad components are given by $A_{1}=A_{3}=1$ and $A_{2}=10$.

The above system is in Hamiltonian form, with $\left(c_{j}, c_{j}^{*}\right), j=1,2$ or 3 , being the canonically conjugate variables. The Hamiltonian form of eqs. (5.1) is

$\frac{\mathrm{d} c_{j}}{\mathrm{~d} t}=\mathrm{i} \omega_{j} \frac{\partial H}{\partial c_{j}^{*}}, \quad j=1,2$ or 3

with the Hamiltonian $H$ given by

$H=\sum_{j} \gamma_{123} \operatorname{Im}\left(c_{1} c_{2} c_{3}\right)=$ constant

where the coefficient $\gamma_{123}$ in eq. (5.3) is given by eq. (3.3). Thus, the dynamical system (5.1) has three degrees of freedom and, at least, three integrals of motion: the Hamiltonian $H, E^{(2)}$ and $P^{(2)}$ (which refer, respectively, to the quadratic part of total energy and pseudo-momentum). Therefore, the three-wave problem is integrable. General solution of this problem can be obtained in terms of Jacobian elliptic functions as in Domaracki and Loesch (1977) and Ripa (1981).

Although the dynamics of the resonant three-wave problem (5.1) is well known (Domaracki and Loesch, 1977; Ripa, 1981, and others), we show here an example of numerical integration of this problem in order to fix ideas and to discuss the implications of this triad dynamics for some aspects of the tropical atmospheric circulation. We believe that the original contribution of this work for the dynamics of resonant triads stems from the results of the five-wave dynamics shown in Section 5.2. The numerical integration scheme of eq. (5.1) assumes that the non-linear terms in the right-hand side of eq. (5.1) are constant forcings during a very short time $2 \Delta t$ so that, given the coefficients $c_{j}, j=1,2$, and 3 , at $t-\Delta t$ and $t$, an analytic solution can easily be obtained at $t+\Delta t$. In this scheme, the non-linear terms are evaluated at the central level $t$. The initial condition used in the numerical simulations is given by

$c_{j}(0)=A_{j} \mathrm{e}^{\mathrm{i} \lambda_{j}}$

where $j=1,2,3$. The initial phases $\lambda j$ are chosen such that $\sum_{j} \lambda_{j}=\pi / 2$, in order to obtain the maximum energy exchange (Domaracki and Loesch, 1977; Loesch and Deininger, 1979).

The example of the numerical integration of the problem (5.1) is displayed in Fig. 1 for the triad constituted of a RW with $k=$ 1 and $n=1$ (mode 1), an EGW with $k=4$ and $n=3$ (mode 2) and a WGW with $k=3$ and $n=3$ (mode 3 ). This triad refers to case 2 of Table 1 . In this triad, mode 2 has the largest absolute frequency and, thus, the maximum coupling coefficient in absolute value. Therefore, this inertio-gravity mode is the most energetically active of the triad, i.e. corresponds to the wave which supplies energy to the other components or gains energy from the other two modes. In this integration, the initial condition is almost all projected onto mode 2 and only a small fraction is projected onto the other components $\left(A_{1}=A_{3}=1\right.$ and $A_{2}=$ 


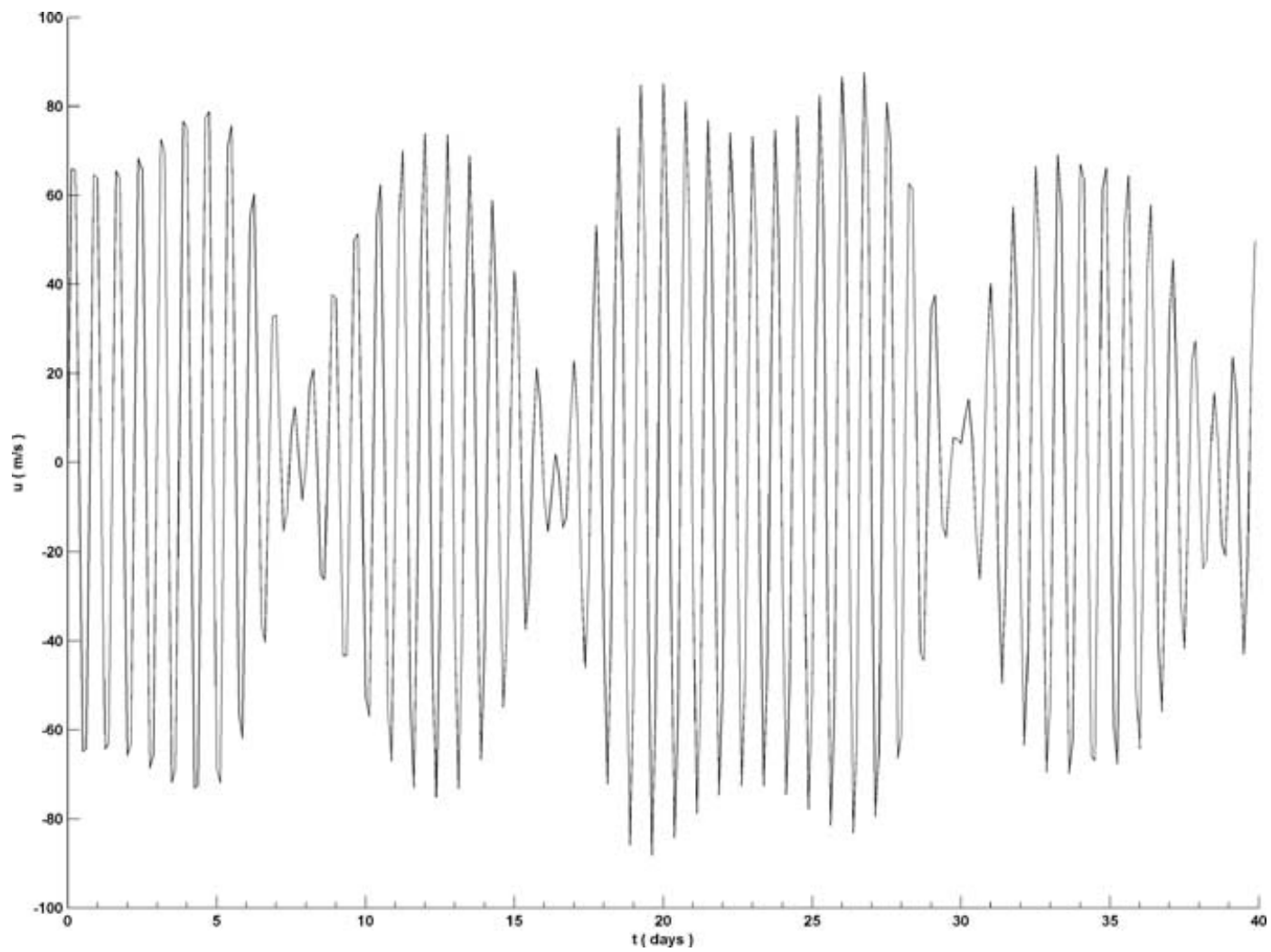

Fig. 2. Time evolution of the zonal component of the velocity field at a certain point of the domain correspondent to the numerical solution of Fig. 1 .

10). Thus, this case is a typical problem of wave instability. As resonant interactions conserve the quadratic part of total energy, the energy of a particular resonant-triad component is simply given by the square of its amplitude.

Figure 1 shows that, when the initial condition is almost totally projected onto the highest-frequency mode, there is a parametric instability of this mode (mode 2), which supplies energy to the components 1 and 3 according to conditions (3.2a) and (3.2b). Nevertheless, after a certain time, mode 2 energy becomes so small that the direction of the energy transfer is reversed, and mode 2 starts to receive energy from the components 1 and 3 . As time evolves, the cycle repeats itself and the energy of each component continues to alternate periodically. Thus, the solution of the resonant three-wave problem is always periodic in time, that is, the triad components exchange energy in a periodic manner while the total energy $E^{(2)}$ remains constant. Some oscillations in the total energy $E^{(2)}$ are observed in Fig. 1 and are due to the fact that the resonance condition is not exactly satisfied, as already discussed.

Another important feature on the triad dynamics involving equatorial waves is that the variation of the RW energy is very small compared to the variation of the gravity wave energies, as expected from eq. (2.5) and from the values of the coupling coefficients (see case 2 of Table 1). Thus, the dynamics of a resonant triad like that of case 2 of Table 1 is characterized by a catalytic energy exchange between the two gravity modes, while the RW energy remains almost unchanged during the process. Therefore, in a resonant triad with two high-frequency modes and one low-frequency mode, the low-frequency mode only acts as a catalytic component. This property implies that the magnitude of the energy modulations is dependent on the distribution of the initial energy among the triad components, as also pointed out by Domaracki and Loesch (1977). In fact, if the initial condition is mostly projected onto mode 1 , the energy exchanges are strongly inhibited (figure not shown).

Another interesting feature regarding the solution of the resonant three-wave problem is that the period of the energy modulations is dependent upon the initial amplitudes but is always finite. Furthermore, the higher the values of the initial amplitudes, the shorter the period of the amplitude modulations (Domaracki and Loesch, 1977).

The important point to be analyzed here refers to the implication of the energy exchanges among resonant triad components for the solution in physical space. Fig. 2 shows the time evolution of the zonal wind field at a certain point of the domain associated with the numerical solution of Fig. 1. The zonal wind field in Fig. 2 was obtained by the expansion (2.3) considering only the three modes of the resonant triad.

The time evolution of the zonal wind field illustrated in Fig. 2 displays the existence of local high-frequency oscillations with a 
period shorter than $1 \mathrm{~d}$. These high-frequency local oscillations are associated with the phase propagation of the two inertiogravity modes of the second triad of Table 1 . In fact, for $h_{0}=$ $250 \mathrm{~m}\left(c=50 \mathrm{~m} \mathrm{~s}^{-1}\right)$, the eastward inertio-gravity wave labeled as mode 2 in Fig. 1, which is characterized by zonal wavenumber 4 and meridional mode 3 , has a time frequency corresponding to a period of the order of $16 \mathrm{~h}$. This short period of the local oscillations may be somewhat unrealistic in the sense that it might not be observed in a more realistic large-scale atmospheric model, which includes a parametrization of the feedback between large-scale dynamics and moist convection. When the interaction between the equatorial wave dynamics and moist convection is taken into account, there is a reduction in the effective equivalent depth of the shallow-water equations and, as a result, there is a reduction in the time frequency of the eigenmodes. In fact, if one assumes that the convective forcing is proportional to divergence, in a first approximation, the divergent term in the continuity equation of model (2.1) appears multiplied by an effective equivalent depth that is smaller than the true equivalent depth. The stronger the convection related to the divergence, the smaller the effective equivalent depth. Therefore, the period of high-frequency oscillations observed in Fig. 2 may reflect the limitation of the model adopted in the present work in not taking into account the interaction between equatorial waves and moist convection. Nevertheless, these high-frequency local oscillations may be, a priori, unimportant for the results presented here, because the focus of the present study is the non-linear interactions. Consequently, we have adopted a phenomenological model with the goal of analyzing only the dynamics of the interaction among equatorial waves through the non-linear terms into the governing equations. The role of reducing the equivalent depth of system (2.1), which implies the incorporation of the interaction between convection and dynamics in the model, on the non-linear dynamics explored here would be an interesting subject for a future work.

The lower-frequency modulation in the amplitude of these local oscillations is resultant from the energy modulations associated with the interactions among the wave modes in the resonant triad. This can be concluded by comparing Figs. 2 and 1. The time when the magnitude of the zonal wind is maximal corresponds exactly to the time when the energy of the EGW (mode 2 ) peaks. On the other hand, when the WGW energy is maximal (and the EGW energy is minimal) corresponds to the time when the amplitude of the zonal wind oscillations is minimal. This is in agreement with the spatial structure of these equatorial waves, because the zonal kinetic energy of the inertio-gravity modes increases as zonal wavenumber increases (shorter waves) (Silva Dias and Schubert, 1979).

Thus, the periodic exchanges of energy among waves constituting a resonant triad imply in periodic changes of regime in the physical space solution. Such changes of regime, in turn, occur on a longer time-scale than the period of the local highfrequency oscillations. This periodic change of regime in the solution in physical space due to the internal dynamics of the model is known as vacillation (Lorentz, 1963). Other mechanisms are also known to induce vacillating solutions, such as wave-zonal flow interactions (Pedlosky, 1977; Boville, 1980) and interference between linear waves with the same wavenumber and different phase speeds (Lindzen et al., 1982). As our results point out, non-linear interactions among waves constituting a resonant triad can also lead to vacillations. The high peak magnitudes of the order of $100 \mathrm{~m} \mathrm{~s}^{-1}$ observed in Fig. 2, which are somewhat unrealistic, are resultant from the unrealistically high value of the mode 2 amplitude set in Fig. 1. We have selected this unrealistic initial wave amplitude in order to more clearly observe the features associated with the dynamics of resonant triads. Exaggerating the high values of the initial amplitudes as done in Fig. 1 makes the dynamical features of the resonant triads, such as the parametric instability of the highest frequency mode, more clearly noticeable. However, it is important to mention that, in a qualitative sense, the results shown in Figs. 1 and 2 are not affected by the unrealistic initial amplitude set in Fig. 1. That is, had we selected lower values for the initial amplitudes in Fig. 1, the behavior of the solution would have been the same as observed in Figs 1 and 2, but the value of the period of the low-frequency amplitude modulation (vacillation) resultant from the energy exchanges would have been different (longer). The only caution that we should have is regarding the conclusions drawn from the periods of the vacillations observed in Fig. 2, as shall be discussed later.

Therefore, the dependence of the period and amplitude of the energy modulation of the modes constituting resonant triads previously discussed have an important implication for the numerical weather prediction. This sensitivity demonstrates that the period of the vacillation cycle of the flow is dependent on the magnitude of the initial field and the way in which this initial field is projected onto the modes that represent the atmospheric flow. The way in which a given state of the atmosphere (which can be used as initial condition in a weather prediction) projects onto the modes, in turn, is very dependent on the manner in which a numerical model represents certain physical process in the atmosphere. For example, gravity waves are directly linked to moist convection. As a consequence, the projection of the initial field onto gravity modes, and consequently the vacillation cycle of the atmospheric flow, can be sensitive to the representation of such moist process in the numerical model, which is highly dependent on the convective parametrization.

\subsection{Problem of two resonant triads coupled by one mode}

In the previous subsection we discussed the dynamics of the energy exchanges among equatorial waves constituting a single resonant triad. Nonetheless, in a more realistic situation, a unique equatorial wave can participate in several resonantly triadic interactions. Thus, in the equatorial wave spectrum are numerous coupled resonant triads, allowing the spectral energy 
redistribution. This shows that resonant interactions can have an important role in the generation of turbulence, making the analysis of the dynamics of resonant triads very useful in understanding the transition to turbulent regimes in dynamical systems. Loesch and Deininger (1979) investigated the effectiveness with which resonant interactions among equatorial waves can redistribute energy generated at selective scales. They analyzed the dynamics of closed systems composed of several resonant triads and pointed out that the energy transference throughout the system is very dependent on the energy projected onto the maximum-frequency modes of individual triads. Here, we explore the dynamics of a more simplified system constituted by only two resonant triads coupled by a unique mode in order to further explore the energy transference between different triads as well as the implications of this process for the tropical atmospheric climate.

Let us consider mode 1 as the mode which couples the two triads. Thus, considering the triads $(1,2,3)$ and $(1,4,5)$, this problem is formulated in terms of the spectral amplitudes as follows:

$$
\begin{aligned}
& \frac{\mathrm{d} c_{1}}{\mathrm{~d} t}-\mathrm{i} \omega_{1} c_{1}=\sigma_{1}^{23} c_{2}^{*} c_{3}^{*}+\sigma_{1}^{45} c_{4}^{*} c_{5}^{*} \\
& \frac{\mathrm{d} c_{2}}{\mathrm{~d} t}-\mathrm{i} \omega_{2} c_{2}=\sigma_{2}^{13} c_{1}^{*} c_{3}^{*} \\
& \frac{\mathrm{d} c_{3}}{\mathrm{~d} t}-\mathrm{i} \omega_{3} c_{3}=\sigma_{3}^{12} c_{1}^{*} c_{2}^{*} \\
& \frac{\mathrm{d} c_{4}}{\mathrm{~d} t}-\mathrm{i} \omega_{4} c_{4}=\sigma_{4}^{15} c_{1}^{*} c_{5}^{*} \\
& \frac{\mathrm{d} c_{5}}{\mathrm{~d} t}-\mathrm{i} \omega_{5} c_{5}=\sigma_{5}^{14} c_{1}^{*} c_{4}^{*} .
\end{aligned}
$$

Problem (5.5) has the same Hamiltonian form given by eq. (5.2) with the Hamiltonian given by

$$
H=\sum_{a b c} \gamma_{a b c} \operatorname{Im}\left(c_{a} c_{b} c_{c}\right)=\text { constant, }
$$

where the subscripts $a, b$ and $c$ range from 1 to 5. However, unlike the three-wave problem, nothing can be said a priori on the integrability of the five-wave system. As in the three-wave problem, the initial condition is given by eq. (5.4) but with $j=$ $1,2,3,4,5$ and the initial phases are chosen in order to obtain the maximum energy exchange.

Examples of numerical solutions of eq. (5.5) are shown in Fig. 3 for the case in which the modes 1,2, 3, 4 and 5 are given by, respectively: an MRGW with $k=5$, an MRGW with $k=1$, an EGW with $k=4$ and $n=1$, a WGW with $k=1$ and $n=2$ and an EGW with $k=4$ and $n=3$. Thus, it can be noted that, in this case, the mode that couples the triads is the lowest-frequency mode in the two triads, i.e. the energetically less active member of the two triads.

Figure $3 \mathrm{a}$ shows the time evolution of the mode energies for the case in which the initial condition is almost projected onto mode 5, which corresponds to the highest-frequency mode of the second triad. As in the three-wave problem, when the initial condition is almost projected onto the highest-frequency mode, there is an initial parametric instability followed by an inversion of the energy transference and a consequent periodic evolution of the energy with time, as shown in Fig. 3a. It is interesting to observe that modes 2 and 3 of the first triad are not significantly excited, that is, their energy is kept suppressed in time. Thus, the solution converges to the three-wave problem and there is no an intertriad energy distribution. The same occurs when the initial condition is equally projected onto the two stable waves of a particular triad, as can be noted in Fig. 3b. In this case, as in Fig. 3a, there is a catalytic energy exchange between the two gravity waves (highest-frequency modes) of the second triad, whereas the MRGW with $k=5$ (which is in the low-frequency category) remains essentially energetically unaltered.

Figure $3 c$ illustrates the case in which all five waves have the same amplitude, i.e. for a 'white noise' initial perturbation. In this case, as observed in Fig. 3c, there are catalytic energy exchanges involving the two highest-frequency modes of each triad. An energy exchange among components of different triads does not occur. In other words, there is no effective intertriad coupling. An interesting feature regarding the solution of system (5.5) occurs when the energy associated with the initial perturbation is equally distributed between two modes of different triads. In this case, as can be seen in Fig. $3 d$ for the case in which the initial perturbation is essentially projected onto components 3 and 5, there are the same catalytic and periodic energy exchanges among modes of the same triad. However, it is clear in the time evolution of the energy of modes 2 and 3 the alternation of large and small energy peaks, suggesting that the period of the energy exchange between these wave components has doubled. This period-doubling seems to be a result of the larger number of degrees of freedom of system (5.5) in comparison to the three-wave problem. The higher the number of triads included in the three-wave problem (5.1), the larger the degrees of freedom of the resulting system, and thus the more irregular the time evolution of the mode energies, tending to a chaotic behavior as the number of degrees of freedom becomes higher. Therefore, the period-doubling of the energy modulation noted in Fig. 3d seems to be an indication of the non-integrability of system (5.5) and, consequently, a possible path to chaos, because the period-doubling rout to chaos is a very well-known example of bifurcation in dynamical systems (Ott, 1993).

Nevertheless, despite the period-doubling of the energy modulations indicating a possible path to chaos as more triads of waves are included to the problem (5.1), the time evolution of the modal energies shown in Fig. 3d also does not display a significant coupling between components of different triads. This absence of an effective intertriad coupling can be due to the fact that the mode that couples the triads is exactly the lowest-frequency mode of the two triads (i.e. the less energetically active member of the system).

In fact, Fig. 4 shows that when the mode coupling the two triads is exactly the highest-frequency mode of the two triads, 
(a)

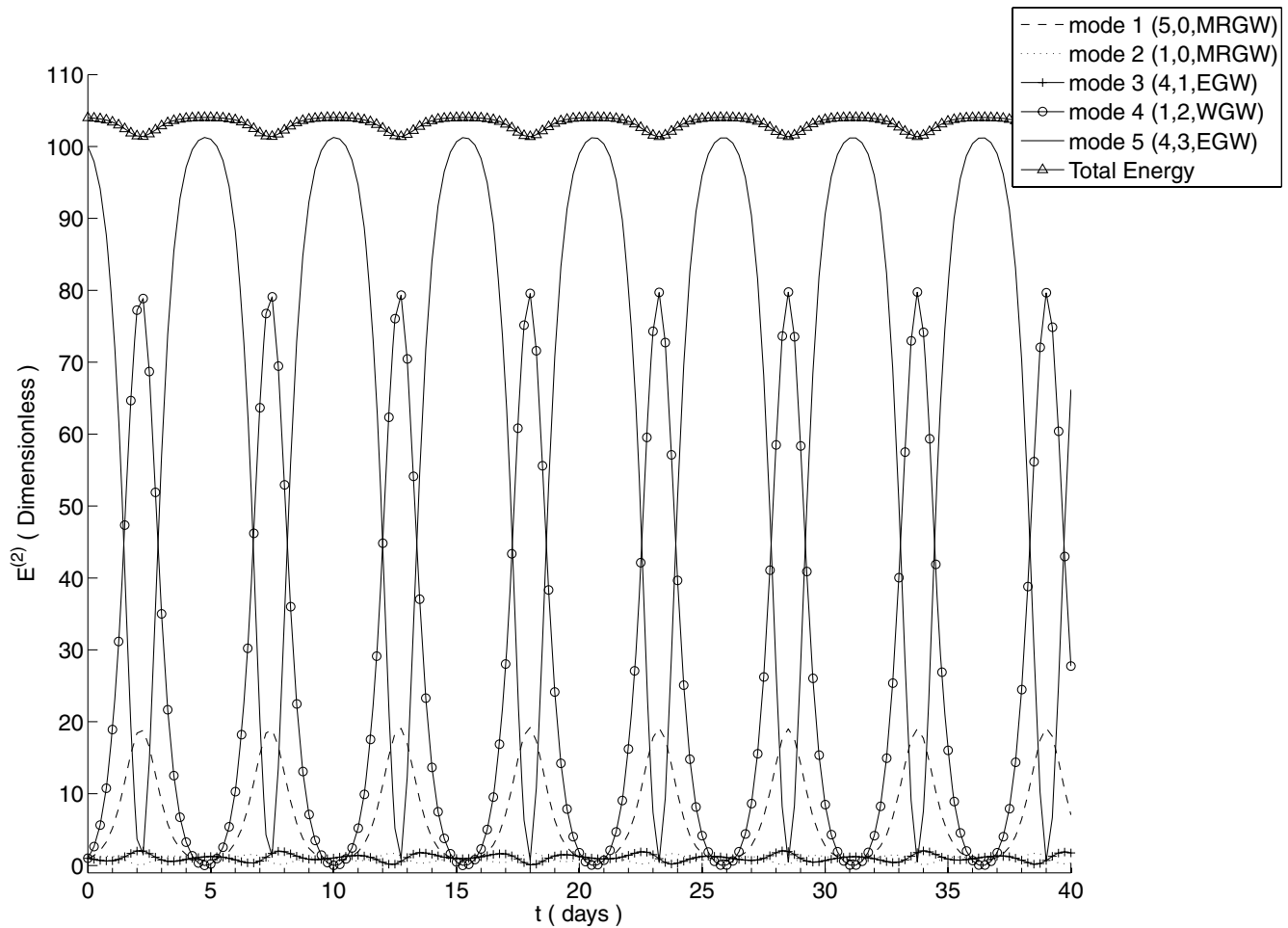

(b)

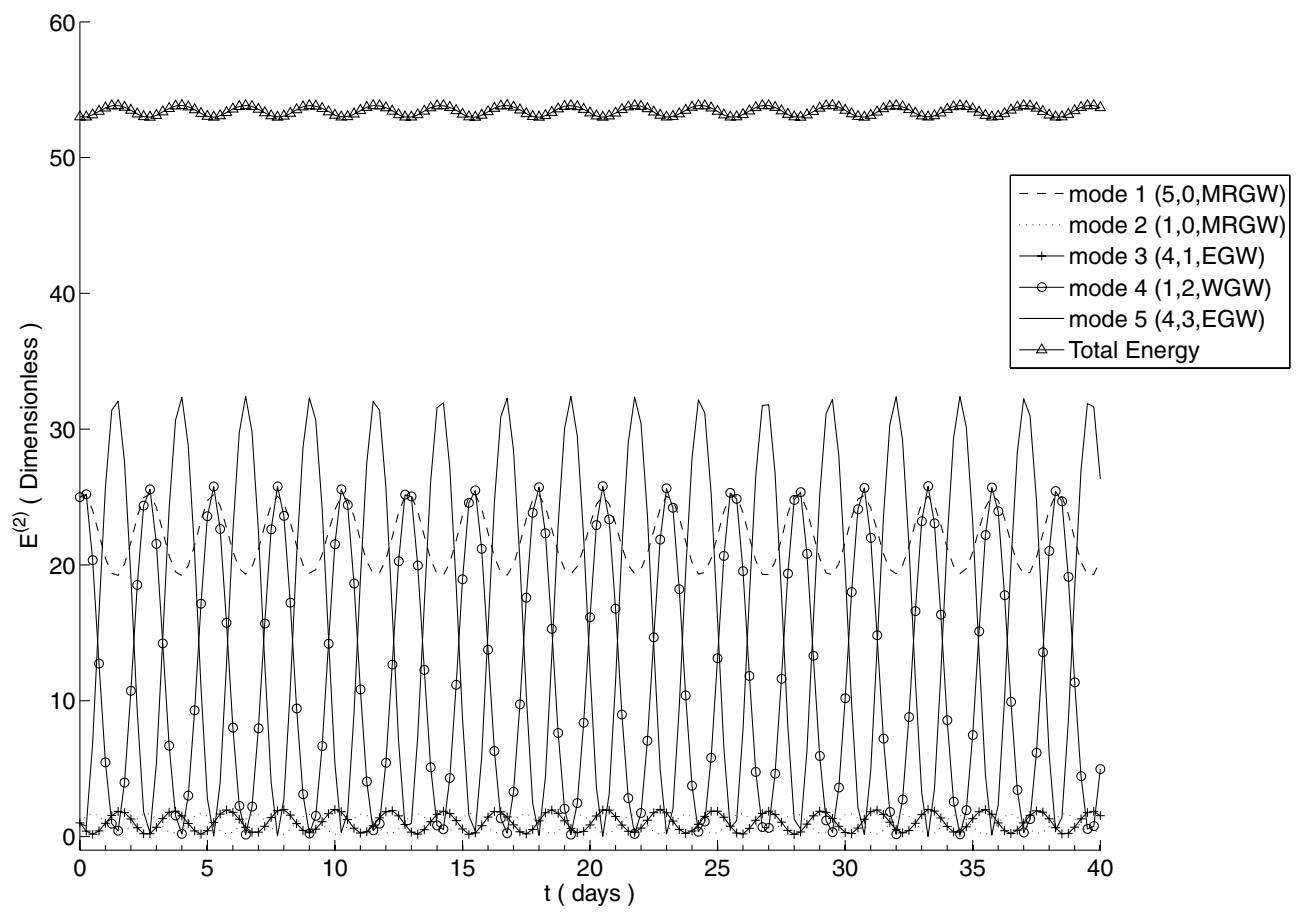

Fig. 3. Time evolution of the modal quadratic energies associated with the solution of problem (5.5) composed of an MRGW with $k=5$ (mode 1), an MRGW with $k=1$ (mode 2), an EGW with $k=4$ and $n=1$ (mode 3), an WGW with $k=1$ and $n=2$ (mode 4 ) and an EGW with $k=4$ and $n=3$ (mode 5). The total quadratic energy given by the sum of the energy of the individual components is also displayed. The initial amplitudes of the modes are given by: (a) $A_{1}=A_{2}=A_{3}=A_{4}=1$ and $A_{5}=10$; (b) $A_{1}=A_{4}=5$ and $A_{2}=A_{3}=A_{5}=1$; (c) $A_{1}=A_{2}=A_{3}=A_{4}=A_{5}=1$; (d) $A_{1}=A_{2}=A_{4}=1$ and $A_{3}=A_{5}=5$. 
(c)

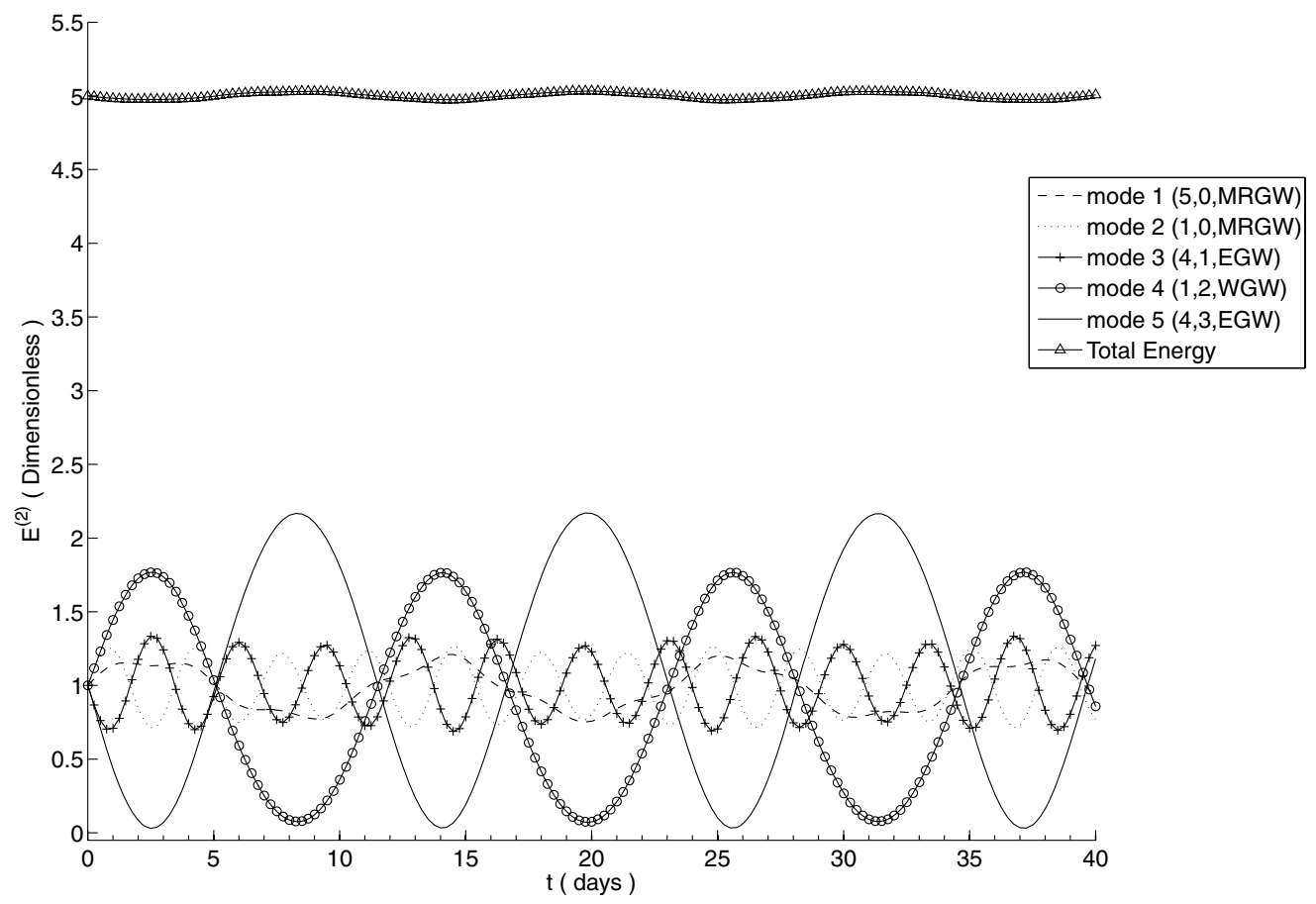

(d)

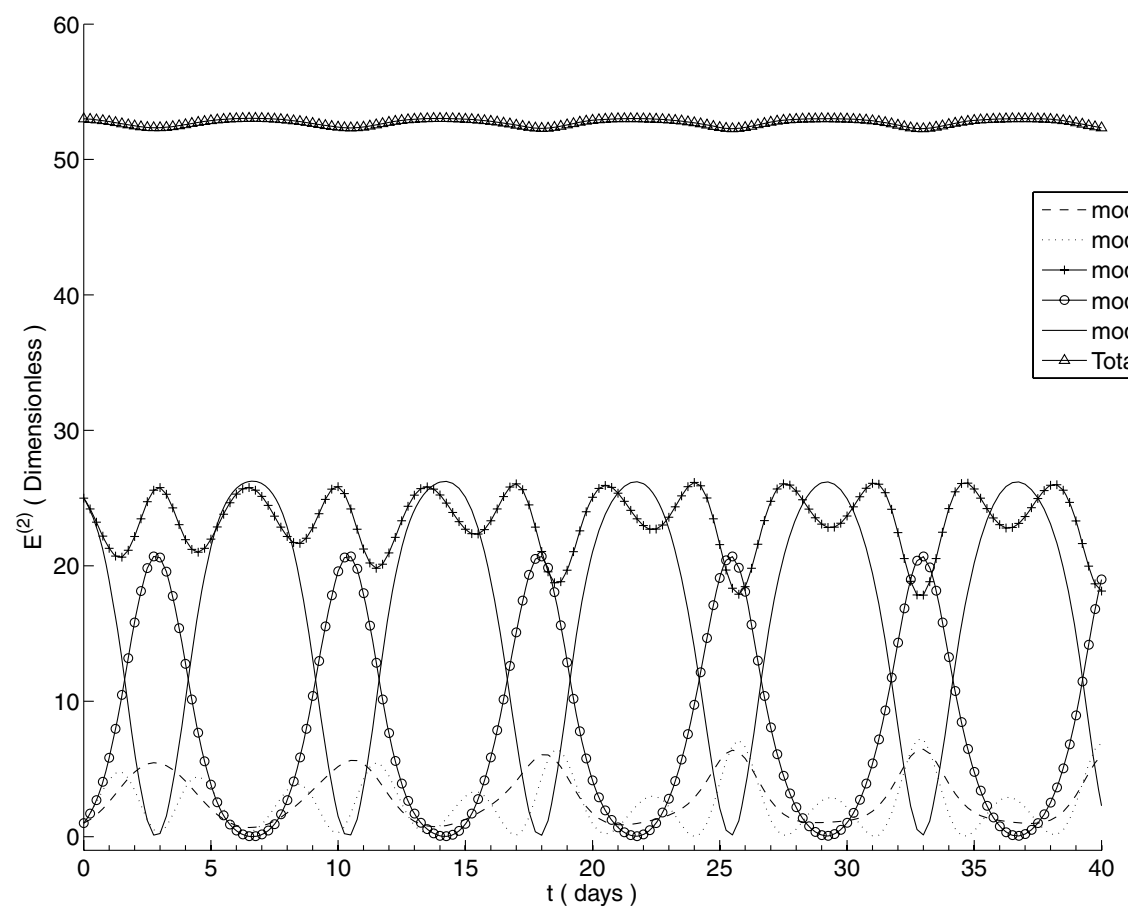

Fig. 3. (cont'd).

a significant intertriad coupling takes place. Figure 4 shows the time evolution of the mode energies associated with the problem (5.5) where the constituting modes are: an EGW with $k=6$ and $n$ $=2($ mode 1$)$; a WGW with $k=6$ and $n=1$ (mode 2$)$; a zonally symmetric MRGW $(k=0)$ ); a WGW with $k=1$ and $n=3$ (mode 4) and an MRGW with $k=7$ (mode 5). It is important to point out that, in this case, the mode which couples the two triads is exactly the highest-frequency mode of the problem. Figure 4a shows the 
(a)

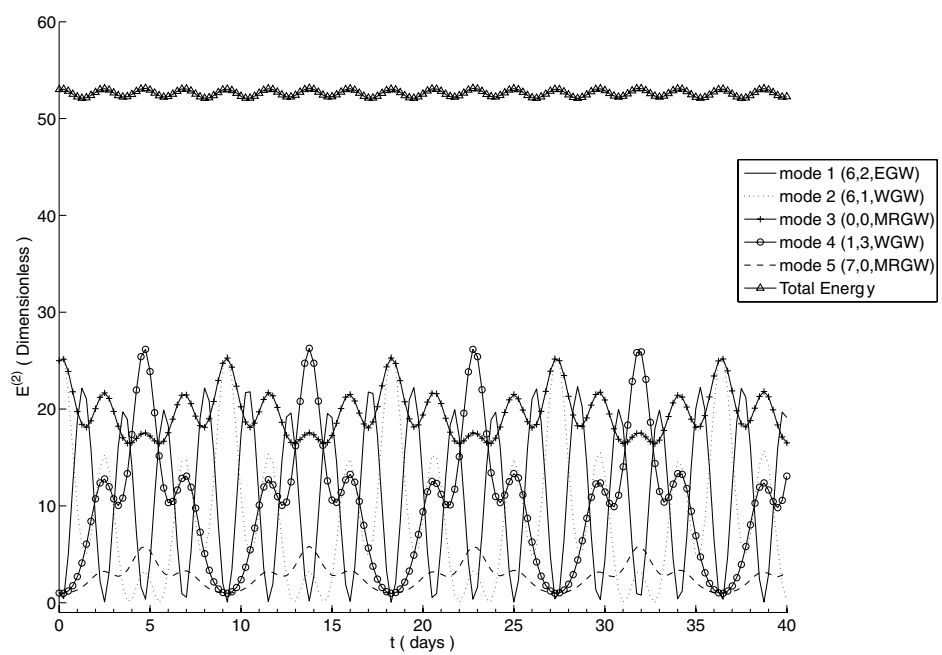

(b)

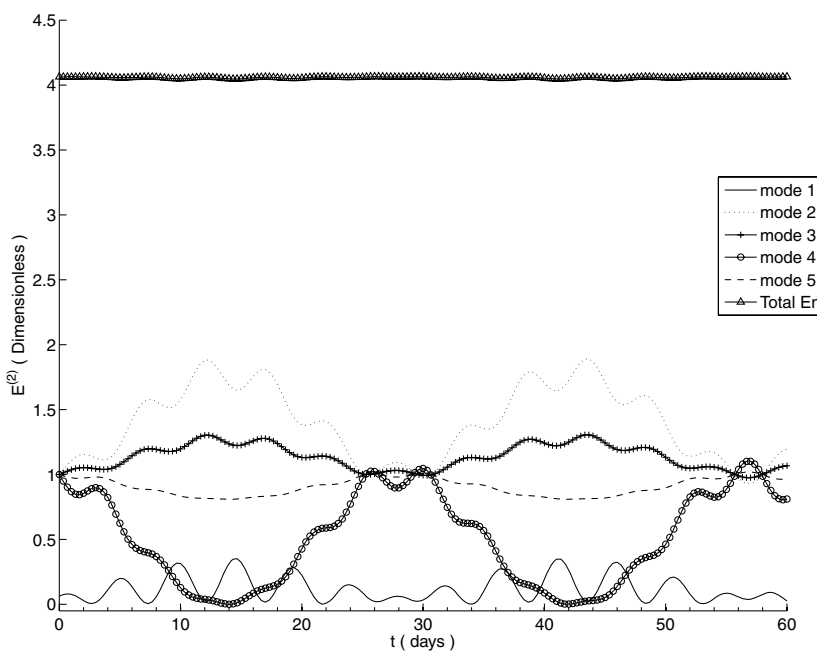

Fig. 4. Similar to Fig. 3, but for the components given by an EGW with $k=6$ and $n=2$ (mode 1$)$, an WGW with $k=6$ and $n=1$ (mode 2), an MRGW with $k=0$ (mode 3), an WGW with $k=1$ and $n=3$ (mode 4) and an MRGW with $k=7$ (mode 5). The initial amplitudes in this case are given by: (a) $A_{2}=A_{3}=5$ and $A_{1}=A_{4}=$ $A_{5}=1$ and (b) $A_{1}=0.25$ and $A_{2}=A_{3}=$ $A_{4}=A_{5}=1$. example in which the initial condition is essentially projected onto the two stable components of the first triad, the modes 2 and 3. Unlike the previous case (Fig. 3b), a significant redistribution of energy occurs, as can be noted by the excitation of mode 4 . It is interesting to note that, besides the energy exchanges among components of the same resonant triad, a noteworthy energy exchange between modes of different triads occurs, as can be seen by the noticeable exchange of energy between modes 4 and 2. Another important feature to be noticed in Fig. 4a is that the period associated with the intertriad energy exchanges is longer than that associated with the intratriad interactions. This longer time-scale of the intertriad energy exchanges is because of the fact that the intertriad coupling is indirect, unlike the intratriad one, as can be seen by eq. (5.5). In order for the mode 2 to supply energy to mode 4 , for example, it has to supply energy to mode 1 at first.

The same intertriad energy exchange observed in Fig. 4a can be more clearly noted in Fig. 4b, which corresponds to the case in which the initial condition is given by a 'white noise' initial perturbation, except that mode 1 has one-quarter of the amplitude of the other modes $\left(A_{1}=0.25 ; A_{2}=A_{3}=A_{4}=\right.$ $A_{5}=1$ ). It is important to observe that the longest period of the energy modulation associated with the intertriad interactions in this case is of order of $30 \mathrm{~d}$ (i.e. even longer than in Fig. 4a).

Figure 5 shows the time evolution of $u$ (zonal component of the wind field) at a certain point of the domain correspondent to the solution of the problem (5.5). Figure 5a corresponds to the numerical solution of Fig. 4b, while Fig. 5 b refers to the same numerical solution as Fig. 5a, but with component 1 having four times the amplitude of the other components $\left(A_{1}=4 ; A_{2}=A_{3}=\right.$ $A_{4}=A_{5}=1$ ). Similarly to the three-wave problem, the $u$ field in Fig. 5 is obtained by the spectral expansion (2.3) truncated in order to consider the five components of problem (5.5). One can note in Fig. 5a three distinct oscillations: a high-frequency oscillation associated with the phase propagation of the 
(a)

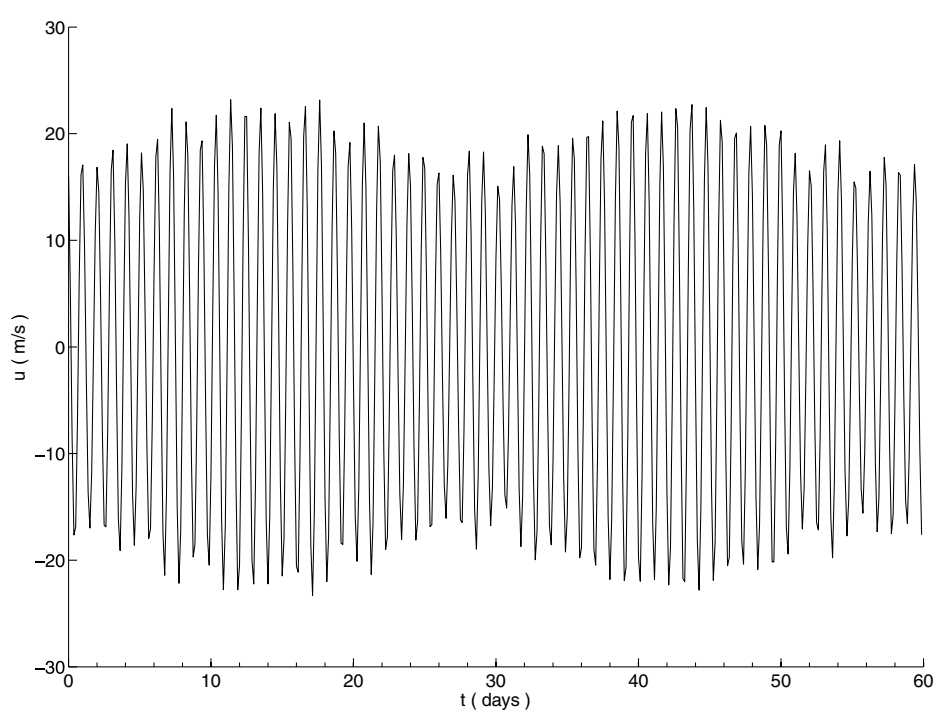

(b)

Fig. 5. Time evolution of the zonal component of the velocity field at a certain point of the domain correspondent to the solution of problem (5.5) with the same mode components as Fig. 4 for (a) $A_{1}=$ 0.25 and $A_{2}=A_{3}=A_{4}=A_{5}=1$ and (b) $A_{1}=4$ and $A_{2}=A_{3}=A_{4}=A_{5}=1$.

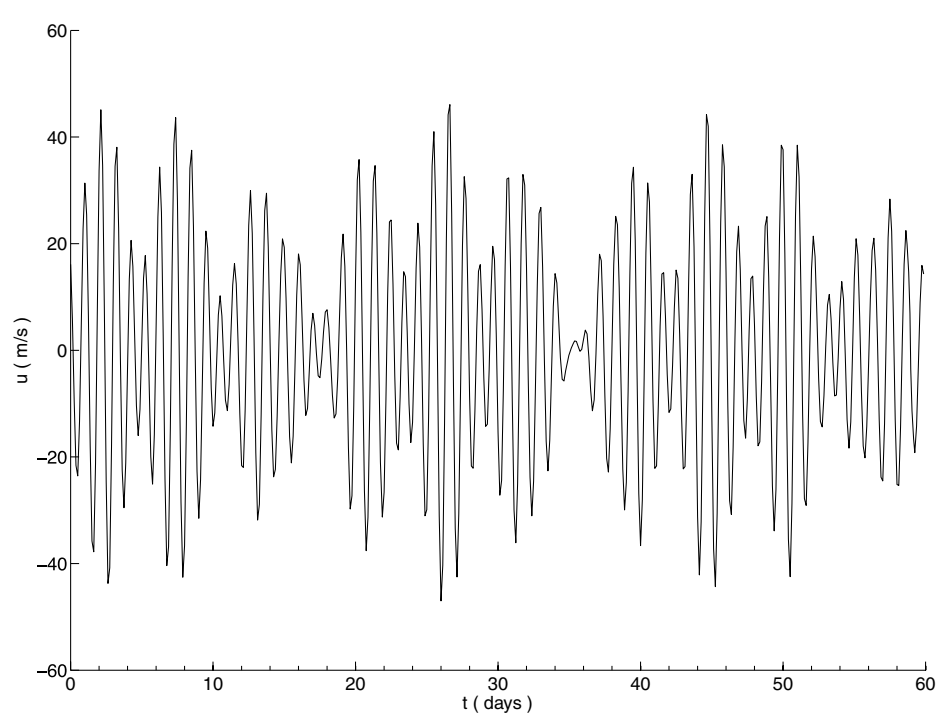

inertio-gravity waves of the two triads; a modulation with longer period (5-8 d), which can be noticed by the alternation of smaller and larger peaks in the high-frequency oscillation's amplitude; and another modulation in the amplitude of the local oscillations with period of the order of $30 \mathrm{~d}$. As discussed in Section 5.1, the short period of high-frequency local oscillations observed in Fig. 5 stems from the absence in our model equations of a convection parametrization. Comparing Figs. $4 \mathrm{~b}$ and $5 \mathrm{a}$ shows that the modulation with intermediate period (5-8 d) is a result of the energy exchanges among modes of the same triad. The same comparison indicates that the longest time-scale modulation, with period of the order of $30 \mathrm{~d}$, is associated with the energy exchanges between modes of different resonant triads (i.e. with the intertriad energy exchanges). Thus, Fig. 5 shows that the inclusion of two modes in the resonant three-wave problem implies in the increase of the vacillation cycles in the physical space solution, with the longest vacillation cycle being resultant from intertriad energy exchanges.

Figure $5 \mathrm{~b}$ indicates that with the amplitude of the highestfrequency mode multiplied by a factor 4 , the same local oscillations and changes of regime occur, but in a shorter period. It can be noted in Fig. 5b that the longest cycle modulation of the local oscillations has a period of the order of $20 \mathrm{~d}$. As in Fig. 2, the unrealistic peak magnitude of the wind field observed in Fig. $5 b$ (of the order of $40 \mathrm{~m} \mathrm{~s}^{-1}$ ) is due to the somewhat unrealistic high initial wave amplitudes set in this numerical simulation $\left(A_{1}=4\right)$. Nevertheless, it is important to state again that this somewhat unrealistic high amplitude has been chosen in order to more clearly notice the dependence of the period of the longest period vacillation resultant from the intertriad interactions upon the initial amplitudes of the wave modes. 
Therefore, the numerical results of the problem of two resonant triads coupled by a single mode discussed in the present item of this section show that a significant interaction between modes of different triads is only possible if the mode coupling the two triads is a high-frequency mode (inertio-gravity or Kelvin mode). Especially, significant intertriad energy exchanges can occur if the mode that couples the triads is the highest-frequency mode of the two triads, as shown in the numerical results of this subsection. In this case, in addition to the interaction among wave components of the same resonant triad, effective energy exchanges involving modes of different triads take place in a longer time-scale than the intratriad ones. As the main energy source of the tropical atmospheric circulation is very scale selective (Kuo, 1975; Lau and Peng, 1987; Wang and Rui, 1990), the results suggest that the high-frequency modes, such as inertiogravity and Kelvin waves, are important in redistributing energy throughout the spectrum of the atmospheric motions and, in turn, in generating large-scale turbulence in the atmospheric flow. As shown in Figs. 4 and 5, for the initial conditions considered here we have obtained periods for the lowest-frequency modulation of the solution of problem (5.5), associated with the intertriad energy exchanges, between 10 and $30 \mathrm{~d}$, depending on the value of the initial energy and the way in which this initial energy is distributed among the wave components. Certainly, had we considered initial conditions with amplitudes smaller than those considered here, these low-frequency modulations associated with intertriad energy exchanges would have undergone periods longer than those observed here. Moreover, in a more realistic model containing more coupled resonant triads, based on the results presented here, there might be modulations with periods even longer. Thus, the results obtained here also suggest that the low-frequency variability of the atmospheric circulation, such as the intraseasonal and/or even longer period variability, can also be associated with non-linear energy exchanges involving modes of different resonant triads. In this process, as shown in the numerical results, the presence of the high-frequency modes coupling the resonant triads is crucial. Thus, the results also suggest the importance of inertio-gravity waves for the low-frequency variability (intraseasonal and/or even longer term) of the atmospheric flow.

\section{Summary and conclusions}

In the present work we have investigated the kinematical and dynamical properties of resonant interactions among equatorial waves in the context of the shallow-water model on the equatorial $\beta$-plane. The spectral method with the basis functions given by the eigensolutions of the linear problem was applied to the governing equations. In this context, from the phase space expansion of some quadratic to lowest-order integrals of motion of the model, some constraints were obtained which the coupling coefficients must satisfy in order to ensure the invariance of such integrals up to third order. From the numerical computa- tion of the coupling coefficients, these constraints were used to determine the possible resonant interactions among equatorial waves. In general, the resonant triads obtained involve all the equatorial wave types and are strongly dominated by triads with two high-frequency modes (Kelvin or inertio-gravity) and one low-frequency mode (Rossby or mixed Rossby-gravity). Some strictly low-frequency and strictly high-frequency triads also exist, but no triads involving solely the inertio-gravity modes could be found. In order to analyze the dynamics of these resonant interactions, the results of numerical integrations of the resonant three-wave problem and the problem of two resonant triads coupled by a single mode have also been discussed. In general, as a consequence of the conservation of the quadratic part of total energy and pseudo-momentum, the resonant triads involving equatorial waves form closed systems in which the mode energies evolve periodically in time, with the period and amplitude of the energy modulations being dependent on the initial condition, that is, dependent on the initial energy and the way in which this energy is distributed among the triad components. From the spectral reconstitution of the physical space solution, the time evolution of this solution shows that the periodic energy exchanges among the triad components imply in periodic changes of regime in the physical space solution. These changes of regime, in turn, occur in a longer time-scale than the period of the local oscillations associated with the phase propagation of the waves. This mechanism provides a possible source of intraseasonal variability in the model atmosphere.

As a consequence of the conservation of the quadratic part of total pseudo-momentum, the coupling coefficients in a resonant triad are proportional to the individual frequencies of the triad components. As a result, in the numerical integrations of both the resonant three-wave problem and the problem of two triads coupled by one mode the high-frequency modes were found to be energetically more active than the low-frequency modes. The latter tend to act as catalytic components in resonant interactions, with the exception occurring for the triads containing only strictly low-frequency modes. The numerical results of the problem of two triads coupled by one mode highlight the importance of the high-frequency modes for the redistribution of energy between different triads. As the main source of energy to the tropical atmosphere is latent heat release, which is very scale selective (Kuo, 1975; Lau and Peng, 1987; Wang and Rui, 1990), the results obtained here suggest the importance of the Kelvin and/or inertio-gravity waves for the redistribution of energy throughout the atmospheric motion spectrum and, consequently, for generating large-scale turbulence in the atmospheric flow.

As also shown by the numerical results of the problem of two resonant triads coupled by one mode, the intertriad energy exchanges provided by the high-frequency modes coupling the two triads occur in a longer time-scale than the intratriad interactions. Thus, the results also suggest that the low-frequency variability of the atmospheric flow, such as the intraseasonal and 
even longer period variability, can also be associated with vacillations resultant from the energy exchanges among modes of different resonant triads. In this process, as pointed out by the numerical results, the role of high-frequency modes is crucial, suggesting the importance of Kelvin and inertio-gravity waves (and therefore of the latent heat release) for the generation of the low-frequency variability of the tropical atmospheric circulation. As the inertio-gravity waves are directly associated with precipitation and moist convection, the results obtained in this paper clearly link the predictability and realistic representation of intraseasonal oscillations in atmospheric models on well founded moist convective parameterization. Dynamical forecast atmospheric models generally exhibit rather poor simulation and low predictability of the Madden-Julian Oscillation (MJO; Chen and Alpert, 1990; Lau and Chan, 1992; Hendon et al., 2000; Jones et al., 2000) and the results presented here propose that such a low skill may be due, at least in part, to the difficulty of the models in representing the interaction between moist convection and large-scale dynamics. An interesting analysis on the theoretical limit of predictability of the MJO by ensembles of twin predictability experiments has been performed by Waliser et al. (2003).

Therefore, considering the results of the problem of two resonant triads coupled by one mode presented in Section 5.2, this paper has also provided an alternative explanation for the origin of the MJO in the tropics. Since the discovery by Madden and Julian over two decades ago (Madden and Julian, 1972), the MJO has continued to be a topic of significant interest due to its clear modulation on the weather conditions and the wide range of phenomena with which it interacts. The main theoretical efforts to physically explain the origin of the MJO and the associated intraseasonal variability of the atmospheric circulation have been basically associated with either the dynamics of convectively coupled Kelvin-Rossby modes or the extratropical forcing mechanism. The former explains the MJO in terms of the linear equatorial Kelvin-Rossby wave dynamics modified by frictional damping and/or coupling with convection (Chang, 1977; Emanuel, 1987; Neelin et al., 1987; Lau and Peng, 1987; Chang and Lim, 1988; Wang, 1988; Wang and Rui, 1990; Moskowitz and Bretherton, 2000). The latter argues that the MJO can be triggered by extratropical Rossby-wave disturbances propagating intermittently toward the equator (Hsu et al., 1990; Kiladis and Weickmann, 1992; Matthews and Kiladis, 1998). The results presented here suggest a possible excitation mechanism in which the intraseasonal variability is associated with vacillations resultant from energy exchanges involving modes of different resonant triads of equatorial waves. In this nonlinear mechanism, as discussed previously, the role of the inertiogravity modes coupling different triads is essential. However, it is important to say that certain caution is necessary in posing the results of this paper as another possible explanation for the origin of the MJO and the associated intraseasonal variability of the tropical atmospheric circulation, because the period of the vacillations resultant from the energy modulations is rather sensitive to the initial wave amplitudes.

Finally, although the present paper displays the potential importance of non-linear mechanisms coupling large-scale equatorial waves in the context of the equatorial shallow-water equations, caution is necessary in interpreting these results for the fully vertically stratified atmosphere, because the Galerkin projection of the non-linear three-dimension primitive equations onto a particular vertical mode does not fall naturally into the non-linear shallow-water equations given by eqs. (2.1) and (2.2), as discussed in Section 2. Moreover, the model adopted in the present work does not consider important physical mechanisms present in the real atmosphere, such as the interaction between moist convection and large-scale dynamics, forcing and dissipation, along with the vertical density stratification. We plan a future study which analyzes the existence of resonant interactions involving the main modes of variability of the large-scale atmospheric circulation (Rossby, inertio-gravity, mixed Rossbygravity and Kelvin modes) in a baroclinic atmospheric model, i.e. associated with different vertical modes. However, the simplified model used here might capture some of the essential ingredients of the more complex atmospheric dynamics.

\section{Acknowledgments}

The work presented in this paper is part of the ongoing research of the $\mathrm{PhD}$ program of the first author developed at University of São Paulo. This PhD program has been financially supported by FAPESP (Fundação de Amparo à Pesquisa do Estado de São Paulo) under GRANT 02/09683-9. The authors also acknowledge the support by the program 'Instituto do Milênio-Avanço Global e Integrado da Matemática Brasileira' (IM-AGIMB). The present work is also part of the research proposed by the PROSUR program, financially supported by the IAI. The authors are also grateful to Dra. Maria Assunção Faus da Silva Dias for the fruitful suggestions to the present paper.

\section{References}

Boville, B. 1980. Amplitude vacillation on an f-plane. J. Atmos. Sci. 37, 1413-1423.

Bretherton, F. P. 1964. Resonant interactions between waves: the case of discrete oscillations. J. Fluid. Mech. 20, 457-479.

Chang, C.-P. 1977. Viscous internal gravity waves and low-frequency oscillations in the tropics. J. Atmos. Sci. 34, 901-910.

Chang, C.-P. and Lim, H. 1988. Kelvin wave Cisk: a possible mechanism for the 30-50 day oscilations. J. Atmos. Sci. 45, 1709-1720.

Charney, J. G. 1971. Geostrophic turbulence. J. Atmos. Sci. 28, $1087-$ 1095.

Chen, T.-C. and Alpert, J. C. 1990. Systematic errors in the annual and intraseasonal variations of the planetary-scale divergent circulation in NMC medium-range forecasts. Mon. Wea. Rev. 118, 2607-2623.

DeMaria, M. 1985. Linear response of a stratified tropical atmosphere to convective forcing. J. Atmos. Sci. 42(18), 1944-1959.

Domaracki, A. and Loesch, A. Z. 1977. Nonlinear interactions among equatorial waves. J. Atmos. Sci. 34, 486-498. 
Dunkerton, T. and Baldwin, M. 1995. Observation of 3-6 day meridional wind oscillations over the tropical Pacific, 1973-1992: horizontal structure and propagation. J. Atmos. Sci. 52(10), 15851601.

Emanuel, K. A. 1987. An air-sea interaction model of intraseasonal oscillations in the tropics. J. Atmos. Sci. 44, 2224-2240.

Gill, A. E. 1980. Some simple solutions for heat-induced tropical circulation. Q.J.R. Meteorol. Soc. 106, 447-462.

Gruber, A. 1974. The wavenumber-frequency spectra of satellite measured brightness in the tropics. J. Atmos. Sci. 31, 1675-1680.

Hendon, H. H., Liebmann, B., Newman, M., Glick, J. D. and Schemm, J. 2000. Medium-range forecast errors associated with active episodes of the Madden-Julian Oscillation. Mon. Wea. Rev. 128, 69-86.

Hirst, A. C. 1986. Unstable and damped equatorial modes in simple coupled ocean-atmosphere models. J. Atmos. Sci. 43, 606-630.

Hsu, H.-H., Hoskins, B. and Jin, F.-F. 1990. The 1985/86 intraseasonal oscillation and the role of the extratropics. J. Atmos. Sci. 47, 823839.

Jones, C., Waliser, D. E., Schemm, J. K. and Lau, W. K. 2000. Prediction skill of the Madden-Julian oscillation in dynamical extended range forecasts. Climate Dyn. 16, 273-289.

Kanamitsu, M., Krishnamurti, T. N. and Depradine, C. 1972. On scale interactions in the tropics during northern summer. J. Atmos. Sci., 29, 698-706.

Kiladis, G. N. and Weickmann, K. M. 1992. Extratropical forcing of tropical Pacific convection during northern winter. Mon. Wea. Rev. 120, 1924-1938.

Kuo, H. L. 1975. Instability theory of large-scale disturbances in the tropics. J. Atmos. Sci. 32, 2229-2245.

Lau, K.-M. 1981. Oscillation in a simple equatorial climate system. J. Atmos. Sci. 38, 248-261.

Lau, K.-M. and Chan, P. H. 1992. Tropical intraseasonal oscillation and its prediction by the NMC operational model. J. Climate. 5, 13651378.

Lau, K.-M. and Lim, H. 1982. Thermally driven motions in an equatorial $\beta$-plane: Hadley and Walker circulations during the winter monsoon. Mon. Wea. Rev. 110, 336-353.

Lau, K.-M. and Peng, L. 1987. Origin of low-frequency (intraseasonal) oscillations in the tropical atmosphere. Part I: basic theory. J. Atmos. Sci. 44, 950-972.

Lim, H. and Chang, C.-P. 1983. Dynamics of teleconnections and walker circulations forced by equatorial heating. J. Atmos. Sci. 40, 18971915.

Lindzen, R. S., Farrell, B. and Jacqmin, D. 1982. Vacillations due to wave interference: applications to the atmosphere and to annulus experiments. J. Atmos. Sci. 39, 14-23.

Loesch, A. Z. and Deininger, R. C. 1979. Dynamics of closed systems of resonantly interacting equatorial waves. J. Atmos. Sci. 36, 1490-1497.

Longuet-Higgins, M. S. and Gill, A. E. 1967. Resonant interactions between planetary waves. Proc. R. Soc. London A 299, 120.

Lorentz, E. N. 1963. The mechanics of vacillation. J. Atmos. Sci. 20, 448-464.

Madden, R. A. and Julian, P. 1972. Description of global-scale circulation cells in the tropics with a 40-50 day period. J. Atmos. Sci. 29, 11091123.

Magaña, V. and Yanai, M. 1995. Mixed Rossby-gravity waves triggered by lateral forcing. J. Atmos. Sci. 52(9), 1473-1486.
Majda, A. J., Rosales, R. R., Tabak, E. and Turner, C. V. 1999. Interaction of long-scale equatorial waves and dispersion of Kelvin waves through topographic resonances. J. Atmos. Sci. 56, 4118-4133.

Maruyama, T. 1967. Large-scale disturbances in the equatorial lower stratosphere. J. Meteorol. Soc. Japan 45, 391-408.

Matsuno, T. 1966. Quasi-geostrophic motions in the equatorial area. J. Meteorol. Soc. Japan 44, 25-43.

Matthews, A. J. and Kiladis, G. N. 1998. The tropical-extratropical interaction between high-frequency transients and the Madden-Julian oscillation. Mon. Wea. Rev. 127, 661-677.

Moskowitz, B. M. and Bretherton, C. S. 2000. An analysis of frictional feedback on a moist equatorial Kelvin mode. J. Atmos. Sci. 57, 21882206.

Neelin, J. D., Held, I. M. and Cook, K. H. 1987. Evaporation-wind feedback and low-frequency variability in the tropical atmosphere. J. Atmos. Sci. 44, 2341-2348.

Ott, E. 1993. Chaos in Dynamical Systems. Cambridge University Press, Cambridge, 385 pp.

Pedlosky, J. 1977. A model of wave amplitude vacillation. J. Atmos. Sci. 34, 1898-1912.

Phillips, O. M. 1960. On the dynamics of unsteady gravity waves of finite amplitude. J. Fluid Mech. 9, 193-217.

Pires, P., Redelsperger, J. L. and Lafore, J. P. 1997. Equatorial atmospheric waves and their association to convection. Mon. Wea. Rev. 6(14), 1167-1184.

Raupp, C. F. M. and Silva Dias, P. L. 2004. Effects of nonlinear process on the interhemispheric energy propagation forced by tropical heat sources. Brazilian J. Meteorol. 19(2), 177-188.

Raupp, C. F. M. and Silva Dias, P. L. 2005. Excitation mechanism of mixed Rossby-gravity waves in the equatorial atmosphere: role of the nonlinear interactions among equatorial waves. J. Atmos. Sci. 62, 1446-1462.

Ripa, P. 1981. On the theory of nonlinear interactions among geophysical waves. J. Fluid Mech. 103, 87-115.

Ripa, P. 1982. Nonlinear wave-wave interactions in a one-layer reducedgravity model on the equatorial $\beta$-plane. J. Phys. Oceanogr. 12, 97111.

Ripa, P. 1983a. Weak interactions of equatorial waves in a one-layer model. Part I: general properties. J. Phys. Oceanogr. 13, 12081226.

Ripa, P. 1983b. Weak interactions of equatorial waves in a one-layer model. Part II: applications. J. Phys. Oceanogr. 13, 1227-1240.

Salmon, R. 1980. Geostrophic turbulence. Proceedings of the Enrico Fermi International School of Physics, Varenna, Italy.

Silva Dias, P. L. and Bonatti, J. P. 1985. A preliminary study of the observed modal structure of the Summer circulation over tropical South America. Tellus 37A, 185-195.

Silva Dias, P. L. and Schubert, W. H. 1979. The dynamics of equatorial mass-flow adjustment. Atmospheric Science Paper No. 312, Department of Atmospheric Science Colorado State University, Fort Collins, CO, USA.

Silva Dias, P. L., Schubert, W. H. and DeMaria, M. 1983. Large-scale response of the tropical atmosphere to transient convection. J. Atmos. Sci. 40, 2689-2707.

Takayabu, Y. 1994. Large-scale cloud disturbance associated with equatorial waves. Part I: spectral features of the cloud disturbances. $J$. Meteorol. Soc. Japan 72, 433-448. 
Waliser, D. E., Lau, K. M., Stern, W. and Jones, C. 2003. Potential predictability of the Madden-Julian oscillation. Bull. Am. Meteorol. Soc. 84(1), 33-50.

Wang, B. 1988. Dynamics of tropical low-frequency waves: an temperatureanalysis of the moist Kelvin wave. J. Atmos. Sci. 45, 2051-2065.

Wang, B. and Rui, H. 1990. Dynamics of the coupled moist KelvinRossby wave on an equatorial $\beta$-plane. J. Atmos. Sci. 47, 397-413.

Webster, P. J. 1972. Response of the tropical atmosphere to local steady forcing. Mon. Wea. Rev. 100, 518-540.
Wheeler, M. and Kiladis, G. N. 1999. Convectively coupled equatorial waves: Analysis of clouds and temperature in the wavenumberfrequency domain. J. Atmos. Sci. 56, 374-399.

Yanai, M. and Maruyama, T. 1966. Stratospheric wave disturbances propagating over the equatorial Pacific. J. Meteorol. Soc. Japan 44, $291-$ 294.

Zangvil, A. and Yanai, M. 1980. Upper tropospheric waves in the tropics. Part 1: dynamical analysis in the wavenumber-frequency domain. J. Atmos. Sci. 37, 283-298. 